\title{
Risk factors for latent tuberculosis infection in close contacts of active tuberculosis patients in South Korea: a prospective cohort study
}

\author{
Seung Jun Lee', Seung Hun Lee ${ }^{1}$, You Eun Kim', Yu Ji Cho ${ }^{1,2}$, Yi Yeong Jeong ${ }^{1,2}$, Ho Cheol Kim ${ }^{1,2^{*}}$,
} Jong Deog Lee ${ }^{1,2}$, Jang Rak Kim²,3, Young Sil Hwang ${ }^{1,2}$, Hee Jin Kim ${ }^{4}$ and Dick Menzies ${ }^{5}$

\begin{abstract}
Background: The diagnosis and treatment of latent tuberculosis infection (LTBI) have become mandatory to reduce the burden of tuberculosis worldwide. Close contacts of active TB patients are at high risk of both active and LTBI. The aim of this study is to identify the predominant risk factors of contracting LTBI, persons in close contact with TB patients were recruited. This study also aimed to compare the efficacy of the tuberculin skin test (TST) and QuantiFERON-TB GOLD (QFT-G) to diagnose LTBI.

Methods: Close contacts of active pulmonary TB patients visiting a hospital in South Korea were diagnosed for LTBI using TST and/or QFT-G. The association of positive TST and/or QFT-G with the following factors was estimated: age, gender, history of Bacillius Calmette-Guerin (BCG) vaccination, history of pulmonary TB, cohabitation status, the acid-fast bacilli smear status, and presence of cough in source cases.
\end{abstract}

Results: Of 308 subjects, 38.0\% (116/305) were TST positive and 28.6\% (59/206) were QFT-G positive. TST positivity was significantly associated with male gender (OR: 1.734; $95 \% \mathrm{Cl}: 1.001-3.003, \mathrm{p}=0.049$ ), history of pulmonary TB (OR: 4.130; 95\% Cl: 1.441-11.835, $p=0.008$ ) and household contact (OR: 2.130; 95\% Cl: 1.198-3.786, $p=0.01$ ) after adjustment for confounding variables. The degree of concordance between TST and QFT-G was fair (70.4\%, $\mathrm{K}=0.392$ ).

Conclusions: A prevalence of $L T B I$ among close contacts of active pulmonary TB patients was high, and prior TB history and being a household contact were risk factors of LTBI in the study population.

Keywords: Tuberculin test, Interferon-gamma release tests, Latent tuberculosis, Independent living, Tuberculosis, pulmonary

\section{Background}

According to the report of World Health Organization (WHO), there are about 8.6 million incident cases of tuberculosis (TB) and about 1.3 million deaths from TB annually [1]. A Stop TB Partnership has been established to reduce the TB burden to less than 100,000 cases per year by 2050 by focusing on areas of high TB burden [2]. However, persons in close contacts with $\mathrm{TB}$ patients are also at a risk of contracting latent TB infection (LTBI).

\footnotetext{
* Correspondence: hochkim@gnu.ac.kr

'Department of Internal Medicine, College of Medicine, Gyeongsang

National University, 90 Chilam-Dong, Jinju, Gyeongnam 660-302, South Korea

${ }^{2}$ Gyeongsang Institute of Health Sciences, Gyeongsang National University,

Jinju, South Korea

Full list of author information is available at the end of the article
}

In 2010, the WHO estimates that nearly $1 / 3$ of the world's population contracted LTBI, and that $10 \%$ of these carriers will develop an active TB infection [3]. The vast majority of these patients will become infectious and perpetuate the cycle of morbidity and mortality. Therefore, it is imperative to diagnose LTBI patients early and efficiently to reduce the global burden of TB.

The traditional method to diagnose TB infection is tuberculin skin test (TST). However, this test does not discriminate LTBI patients. The FDA recently approved the use of interferon gamma release assays (IGRAs) such as the QuantiFERON-TB Gold ${ }^{\bullet}$ (QFT-G) assay for the diagnosis of LTBI [4]. In 2011, Korean guidelines stipulate that IGRAs are an alternative method of TST for the 
diagnosis of LTBI [5]. IGRAs have some advantages over TST. First, IGRAs lack booster effect. Second, BCG vaccination and infection by non-tuberculosis mycobacterium (NTM) has less effect on the IGRA than on the TST. Third, the patients don't need to revisit for reading the results [6]. There are several risk factors known to be associated with developing active TB in close contacts, including malnutrition, untreated LTBI, being a household contact, age under 5 years, acid-fast bacilli (AFB) positivity of source case, concomitant human immunodeficiency virus infection, and immunocompromised status $[7,8]$. However, there is limited data about risk factors for LTBI in close contacts.

The present study was conducted to identify the predominant risk factors of contracting the latent form of the disease and to compare the efficiency of the TST and QFT-G to diagnose LTBI subjects in close contacts of active pulmonary $\mathrm{TB}$ patients.

\section{Methods}

\section{Subjects and study design}

All data presented in this study were acquired during an international multi-center study (title: A Randomized Clinical Trial Comparing 4RIF vs. 9INH for Treatment of Latent Tuberculosis Infection - Effectiveness, Registration number; NCT00931736). The primary objective of the multi-center study is to compare the effectiveness of two regimens in preventing active tuberculosis. Full details of the original study are provided in the study protocol, see Section 2 in the Additional file 1: Appendix. In this study, close contacts of culture-positive pulmonary TB patients who visited Gyeongsang National University Hospital, Jinju, South Korea, were prospectively enrolled between October 2009 and August 2012. Close contacts are recommended to visit a hospital because screening of close contacts is a national policy project in South Korea. The current study was approved by the Gyeongsang National University Hospital Institutional Review Board (approval number IRB-2009-042). Close contact was defined as exposure to AFB smear-positive and/or culturepositive active pulmonary TB patient during their infectious period for at least 4 hours per week. The duration of exposure was determined by self-reported questionnaire. Household contacts were defined as those living in the same house with source case. Exclusion criterion was active TB identified by chest X-ray. The remaining subjects filled informed consent documents and were tested for LTBI by TST and/or QFT-G. Both tests were conducted on the same day or TST was conducted first. We also documented seven potential risk factors for LTBI: age, gender, history of Bacillus Calmette-Guerin (BCG) vaccination, history of pulmonary TB, living in the same house with a TB patient, the presence of cough, and AFB smear status of the source case. By interviewing enrolled subjects and source patients, age, gender, history of tuberculosis in the close contacts and cough symptom of source case were recorded. History of BCG vaccination was verified by BCG scar. The previous history of TB was identified by self-reported questionnaire. Level of contact was categorized into household or non-household.

\section{TST and QFT-G}

TST was conducted by Mantoux method using purified protein derivative (PPD, Statens Serum Institute; Copenhagen, Denmark). The subjects were given an intradermal injection of Tuberculin ( 2 units in $0.1 \mathrm{ml}$ ) on the forearm. Transverse diameter of induration was measured and recorded in millimeters between 48 and 72 hours. TST was considered positive when induration size was equal to or greater than $5 \mathrm{~mm}$ according to the cut-off value of the multi-center study. Induration was read by skilled and trained physicians. QFT-G test (Cellestis Ltd, Carnegie, VIC, Australia) was carried out according to the manufacturer's instructions. Briefly, whole blood was collected and aliquots were incubated for 16 to 24 hours with TB-specific antigens, including early secretory antigenic target 6-kda protein (ESAT-6) and culture filtrate protein 10 (CFP-10). The concentration of released IFN- $\gamma$ was measured by enzyme-linked immunosorbent assay (ELISA) $[9,10]$.

\section{Statistical analysis}

Descriptive statistics were used to assess the correlation between the seven potential risk factors and TST and/or QFT-G positivity. Each variable was analyzed using a Pearson's Chi-square test. Risk of having LTBI for each potential risk factor was estimated using multinomial logistic regression. Kappa ( $\mathrm{k}$ ) statistics were used to measure concordance between TST and QFT-G and classified as follows: $\mathrm{K} \leq 0=$ no agreement; $0<\mathrm{K} \leq 0.2=$ poor agreement; $0.2<\mathrm{K} \leq 0.4=$ fair agreement; $0.4<\mathrm{\kappa} \leq 0.6=$ moderate agreement; $0.6<\mathrm{k} \leq 0.8=$ substantial agreement; $0.8<\mathrm{K} \leq 1.0=$ optimal agreement. Differences were considered statistically significant if $\mathrm{p}<0.05$. Statistical analyses were performed using SPSS version 18.0 for Window (SPSS Inc, Chicago, Illinois, USA).

\section{Results}

\section{LTBI in the study population}

The review of 129 cases of active TB patients identified a total of 308 close contact individuals (103 males and 205 females) confirmed not to have active TB by chest Xrays (Table 1). Subjects were household or work contacts of patients with confirmed active pulmonary TB. TST was administered and read for 305 subjects of whom 116 (38.0\%) had a positive result. QFT-G was carried out in 206 subjects of whom 59 (28.6\%) showed a positive result. Both tests were completed in 203 subjects of 
Table 1 Baseline characteristics of the study population

\begin{tabular}{|c|c|c|}
\hline & $\begin{array}{l}\text { TST } \\
\text { participants } \\
(\mathrm{n}=305)\end{array}$ & $\begin{array}{l}\text { QFT-G } \\
\text { participants } \\
(\mathrm{n}=206)\end{array}$ \\
\hline \multicolumn{3}{|l|}{ Demographics } \\
\hline Male & $103(33.8 \%)$ & $66(32.0 \%)$ \\
\hline Age (Mean \pm SD) & $44.2 \pm 15.1$ & $43.7 \pm 14.7$ \\
\hline $15-24$ & $28(9.2 \%)$ & $19(9.2 \%)$ \\
\hline $25-34$ & $61(20.0 \%)$ & $42(20.4 \%)$ \\
\hline $35-44$ & $75(24.6 \%)$ & $52(25.2 \%)$ \\
\hline $45-54$ & $72(23.6 \%)$ & $48(23.3 \%)$ \\
\hline $55-64$ & $41(13.4 \%)$ & $29(14.1 \%)$ \\
\hline$\geq 65$ & $28(9.2 \%)$ & $16(7.8 \%)$ \\
\hline \multicolumn{3}{|l|}{ Household contact } \\
\hline Yes & $164(53.8 \%)$ & $105(51.0 \%)$ \\
\hline \multicolumn{3}{|l|}{ Test result } \\
\hline Positive & $116(38.0 \%)$ & 59 (28.6\%) \\
\hline \multicolumn{3}{|c|}{ BCG vaccination scar } \\
\hline Yes & $288(94.4 \%)$ & $194(94.2 \%)$ \\
\hline \multicolumn{3}{|c|}{ History of pulmonary TB } \\
\hline Yes & $22(7.2 \%)$ & $15(7.3 \%)$ \\
\hline \multicolumn{3}{|c|}{$\begin{array}{l}\text { AFB smear status of source } \\
\text { cases }\end{array}$} \\
\hline Positive & $236(77.4 \%)$ & $152(73.8 \%)$ \\
\hline \multicolumn{3}{|c|}{ Cough of source cases } \\
\hline Yes & $184(60.3 \%)$ & $121(58.7 \%)$ \\
\hline
\end{tabular}

TB Tuberculosis, BCG Bacillus of Calmette-Guerin, TST Tuberculin skin test, QFT-G QuantiFERON` TB GOLD.

whom 138 subjects underwent both tests on the same day. QFT-G was conducted later in 65 subjects and median interval between both tests was 3 days. More than $90 \%$ of the subjects had a BCG vaccination $(94.4 \%$ in the TST group, $94.2 \%$ in the QFT-G group). A total of 7.2\% subjects from the TST group and $7.3 \%$ subjects from the QFT-G group had a history of previous active pulmonary TB. AFB staining of smear of source cases was positive in $77.4 \%$ in the TST group and $73.8 \%$ in the QFT-G group. A total of $53.8 \%$ subjects in the TST group and $51.0 \%$ subjects in the QFT-G group were household contacts. Presence of cough in source cases was $60.3 \%$ in the TST group and $58.7 \%$ in the QFT-G group (Table 1). Comorbid conditions included hypertension in 21 , diabetes in 8 , cardiovascular disease in 7 , thyroid disease in 7, chronic liver disease in 5 subjects (data not shown).

\section{Risk factors for LTBI}

The three potential risk factors that were not associated with TST- or QFT-positivity were as follows: history of
BCG vaccination, cough and AFB smear status of source case (Table 2). Age was associated with TST positivity when stratified into 10-year old, but not with QFT-G positivity. Occurrence of positive TST was significantly higher in males than in females, but there was no significant difference in the occurrence of positive QFT-G between males and females. Both TST- and QFT-positivity were significantly associated with being a household contact. Subjects with history of TB showed significantly higher TST and QFT-G positivity than those without TB (Table 2).

Multivariate logistic regression was performed with either TST- or QFT-positivity as the dependent variable and the seven risk factors as independent variables (Table 3).

Table 2 Association of seven potential risk factors with TST or QFT-G positivity

\begin{tabular}{|c|c|c|c|c|}
\hline & $\begin{array}{l}\text { TST positive, } \\
\text { n (\%) }\end{array}$ & $p$ value & $\begin{array}{l}\text { QFT-G positive, } \\
\text { n (\%) }\end{array}$ & $\mathrm{p}$ value \\
\hline Age & & $<0.001$ & & 0.063 \\
\hline $15-24$ & $2(7.1)$ & & $2(10.5)$ & \\
\hline $25-34$ & $16(26.2)$ & & $6(14.3)$ & \\
\hline $35-44$ & $39(52.0)$ & & 19 (36.5) & \\
\hline $45-54$ & $34(47.2)$ & & $16(33.3)$ & \\
\hline $55-64$ & $16(39.0)$ & & $10(34.5)$ & \\
\hline$\geq 65$ & $9(32.1)$ & & $6(37.5)$ & \\
\hline Gender & & 0.001 & & 0.176 \\
\hline Male & $53(51.5)$ & & $23(34.8)$ & \\
\hline Female & $63(31.2)$ & & $36(25.7)$ & \\
\hline $\begin{array}{l}\text { BCG vaccination } \\
\text { scar }\end{array}$ & & 0.430 & & 0.092 \\
\hline Yes & $108(37.5)$ & & $53(27.3)$ & \\
\hline No & $8(47.1)$ & & $6(50.0)$ & \\
\hline Household contact & & 0.023 & & 0.033 \\
\hline Yes & $72(43.9)$ & & $37(35.2)$ & \\
\hline No & $44(31.2)$ & & $22(21.8)$ & \\
\hline $\begin{array}{l}\text { History of } \\
\text { pulmonary TB }\end{array}$ & & 0.001 & & 0.028 \\
\hline Yes & $16(72.7)$ & & $8(53.3)$ & \\
\hline No & $100(35.3)$ & & $51(26.7)$ & \\
\hline $\begin{array}{l}\text { Smear status of } \\
\text { source cases }\end{array}$ & & 0.139 & & 0.118 \\
\hline Positive & $95(40.3)$ & & 48 (31.6) & \\
\hline Negative & $21(30.4)$ & & $11(20.4)$ & \\
\hline $\begin{array}{l}\text { Cough of source } \\
\text { cases }\end{array}$ & & 0.053 & & 0.094 \\
\hline Yes & $78(42.4)$ & & $40(33.1)$ & \\
\hline No & $38(31.4)$ & & $19(22.4)$ & \\
\hline
\end{tabular}

TB Tuberculosis, BCG Bacillus of Calmette-Guerin, TST Tuberculin skin test, QFT-G QuantiFERON`-TB GOLD. 
Table 3 Multivariate analysis between seven risk factors and TST or QFT-G positivity

\begin{tabular}{|c|c|c|c|c|}
\hline & TST po & & QFT-G p & \\
\hline & Odds ratio $(95 \% \mathrm{Cl})$ & $p$ value & Odds ratio $(95 \% \mathrm{Cl})$ & $p$ value \\
\hline Gender (male) & $1.734(1.001-3.003)$ & 0.049 & $0.982(0.476-2.025)$ & 0.961 \\
\hline Age & & & & \\
\hline $15-24$ & 1 & & 1 & \\
\hline $25-34$ & $6.373(1.273-31.909)$ & 0.024 & $1.495(0.267-8.360)$ & 0.647 \\
\hline $35-44$ & $13.769(2.865-66.172)$ & 0.001 & $4.103(0.809-20.820)$ & 0.088 \\
\hline $45-54$ & $10.552(2.221-50.141)$ & 0.003 & $3.081(0.607-15.625)$ & 0.174 \\
\hline $55-64$ & $6.597(1.307-33.284)$ & 0.022 & $3.070(0.555-16.975)$ & 0.199 \\
\hline$\geq 65$ & $2.089(0.284-15.379)$ & 0.470 & $1.340(0.134-13.355)$ & 0.803 \\
\hline BCG vaccination scar (yes) & $0.335(0.067-1.681)$ & 0.184 & $0.361(0.055-2.370)$ & 0.289 \\
\hline History of pulmonary TB (yes) & $4.130(1.441-11.835)$ & 0.008 & $2.561(0.819-8.002)$ & 0.106 \\
\hline Household contact (yes) & $2.130(1.198-3.786)$ & 0.010 & $1.911(0.920-3.971)$ & 0.083 \\
\hline Smear status of source case (positive) & $1.460(0.745-2.864)$ & 0.271 & $1.947(0.831-4.564)$ & 0.125 \\
\hline Cough of source case (yes) & $1.324(0.753-2.328)$ & 0.329 & $1.167(0.576-2.362)$ & 0.669 \\
\hline
\end{tabular}

TB Tuberculosis, BCG Bacillus of Calmette-Guerin, TST Tuberculin skin test, QFT-G QuantiFERON-TB GOLD, CI Confidence interval.

Male gender, history of pulmonary TB and household contact significantly increased odds ratio of TST positivity (Table 3). There was definitely no gender difference in QFT-G positivity, but there was a trend toward QFT-G positivity associated with history of pulmonary $\mathrm{TB}$ and household contact. These results suggest that prior TB history and being a household contact are the predominant risk factors for LTBI.

\section{Concordance between TST and QFT-G}

Among total of 308 participants, 305 subjects performed TST and 206 subjects performed QFT-G testing. Twohundred-three subjects completed both TST and QFT-G testing. Among them, 46 subjects were positive for both TST and QFT-G, 49 subjects were TST positive but QFTG negative, 11 subjects were QFT-G positive but TST negative, and 97 subjects were negative for both tests. Concordance rate of two tests was $70.4 \%$. The kappa value showed fair agreement $(\kappa=0.392)$, but showed a significant correlation between two tests $(\mathrm{p}<0.001$, Table 4$)$.

\section{Discussion}

This study showed that positive rate of TST and QFT-G is high in those who are in close contact with active

Table 4 Correlation between TST and QFT-G ( $n=203)$

\begin{tabular}{|c|c|c|c|c|c|c|}
\hline & & \multicolumn{2}{|c|}{ QFT-G } & \multirow[b]{2}{*}{ K } & \multirow[b]{2}{*}{$p$ value } & \multirow[b]{2}{*}{ Agreement, \% } \\
\hline & & Positive & Negative & & & \\
\hline \multirow[t]{2}{*}{ TST } & Positive & 46 & 49 & & & \\
\hline & Negative & 11 & 97 & 0.392 & $<0.001$ & 70.4 \\
\hline
\end{tabular}

TST Tuberculin skin test, QFT-G QuantiFERON-TB GOLD. pulmonary TB patients. History of TB and household contact were significantly associated with the risk of TST positivity and concordance between TST and QFT$\mathrm{G}$ was $70.4 \%$.

The prevalence of LTBI is expected to be high in close contacts of active TB patients. The prevalence of LTBI in close contacts was reported to be highest among many risk groups for LTBI, including close contacts, foreignborn people, homeless people, injection drug users, and prisoners [11]. Furthermore, close contact is one of the common risk factor for progression from LTBI to active disease [11]. In the present study, the positive rates of TST and QFT-G were $38.0 \%$ and $28.6 \%$ respectively, in South Korean population. Marks et al. reported that a prevalence of LTBI is about $36 \%$ in close contacts of persons with infectious TB as assessed by TST in a study conducted in the United States [12]. Meanwhile, the prevalence of LTBI in household contacts in countries with a high burden of TB is diversely reported from $27 \%$ to $93 \%$, again based on TST [13]. The positive rate of QFT-G in contact investigation for TB was $30.2 \%$ in Germany and $19 \%$ in Taiwan [14,15]. Given that South Korea has an intermediate burden of TB cases and almost every child receives $B C G$ vaccination at birth, the positive rates of both tests in this study, especially of QFT-G, were considerably high. In a previous multi-center study conducted in South Korea, the positive rate of QFT-G in health care workers was $17.2 \%$ and that of TST was $36.7 \%$ [16]. Because QFT-G is not affected by the previous BCG vaccination and most of the NTM infection, high positive rate of QFT-G in this study reconfirms the incidence of LTBI in close contacts of active TB patients is quite high in South Korean population. 
Increasing age was significantly associated with TST positivity, but not with QFT-G positivity in the current study. Li et al. reported that prevalence of TST positivity significantly increased with age [17]. Furthermore, according to the reports of Pareek et al. and Shanaube et al., the result of QFT-G as well as that of TST was associated with increasing age $[18,19]$. It is uncertain whether increased age is the risk factor of contracting LTBI or whether increased cumulative exposure to Mycobacterium tuberculosis and/or NTM as people grow older increases positivity of both tests.

Active $\mathrm{TB}$ is reported to be more prevalent in men than in women, but reasons for this phenomenon are uncertain due to the lack of studies about gender differences in patients with TB. Gender differences in patients with LTBI were also not studied much. In our study, male gender significantly increased relative risk of TST positivity but not of QFT-G positivity. More data will be needed to clarify the influence of gender in the incidence of LTBI.

The occurrence of LTBI in close contacts with previous pulmonary TB history was significantly high in this study. We aimed to assess the association between previous history of pulmonary TB and the risk of contracting LTBI because the impact of previous history of pulmonary TB on the LTBI had not been well studied. TST and QFT-G are methods to measure lasting adaptive immune responses to Mycobacterium tuberculosis rather than to identify LTBI directly [20]. Direct identification of LTBI was impossible until recently. Although IGRA is a officially approved test for the diagnosis of LTBI, Kim et al. reported that IGRA has a limited role in the diagnosis of TB infection in individuals with a history of TB [21]. Thus, it is uncertain whether positive results of TST and QFT-G depend on the presence of living mycobacteria or persistent immune responses of previous TB infection. Nevertheless, close contacts of active TB patients are at a high risk of infection, and QFT-G is suggested to detect INF- $\gamma$ released by effector T-cells rather than memory T-cells within a short period of incubation $[22,23]$. It is therefore mandatory to study the effects of previous TB history on LTBI, including through clinical trials, and to understand the molecular basis of T-cell responses.

Household contact increased the risk of TST- and IGRA-positivity in previous studies [24,25]. Likewise, the result of current study showed that household contact significantly increased relative risk of being TST positive and QFT-G was tend to be more positive in household contacts. It is therefore reasonable to pay particular attention to household contacts during the contact investigation of active TB.

The agreement and kappa values of concordance of TST and IGRA have been reported widely in TB contacts
$[9,15,26]$. The common outcome of those studies is that BCG vaccination lowered the agreement between the two tests. In one study performed in Denmark, in which about two-thirds of enrolled subjects were not vaccinated with BCG, the agreement between TST and QFT-G was 94\% ( $\mathrm{K}=0.866)$ [9]. In contrast, most of the people in Korea receive $\mathrm{BCG}$ vaccine, and the agreement of two tests in this study was much lower $(70.4 \%, \mathrm{k}=0.392)$. In this study, only 11 of 203 subjects who received both tests were not vaccinated with BCG. Thus, subgroup analysis for non-BCG vaccinated people did not show significant results (data not shown). Additionally, high rate of BCG vaccination lowered the statistical power to assess the impact of BCG vaccination on TST or QFT-G. In this study, 11 subjects were QFT-G positive but TST negative. The result of QFT-G might be false positive even though we could not verify the baseline result of QFT-G before contact to active TB patients. Performing QFT-G test repeatedly will be helpful to clarify this issue.

While designing this study, we assumed that smear status and presence of cough in source cases might be important factors influencing the occurrence of LTBI. However, our results show that none of these were risk factors. Therefore, the limitations of this study are as follows. First, the number of enrolled subjects is relatively small because people in Korea are not aware of the concept of LTBI and the importance of screening contacts of TB patients. The knowledge about LTBI needs to be spread among the Korean people. Second, of the 308 enrolled subjects, only 203 subjects were willing to take both TST and QFT-G tests, because we gave them an option of choosing between TST and/or QFT-G according to the Korean guidelines. Both single screening strategy using TST or IGRA and dual screening strategy using TST and subsequent IGRA were recommended in the Korean guidelines. Third, in 65 among 203 subjects who took both tests, QFT-G was conducted later than TST and in 28 among those 65 subjects, the interval between two tests was over 4 days. Van Zyl-Smit et al. have reported that a booster effect of PPD was evident by day 7 post-TST, but not by day 3 [27]. The booster effect of PPD might have caused false-positive results of QFT-G in some of the subjects in this study. Fourth, the level of contact was not categorized in depth. In particular, in subjects who were a household contact, whether they lived in the same room or not would be an important factor influencing their LTBI risk.

\section{Conclusions}

LTBI is prevalent in close contacts of active pulmonary TB patients in a South Korean population, and history of pulmonary $\mathrm{TB}$ and being a household contact are predominant risk factors of contracting LTBI in this study. 


\section{Additional file}

\section{Additional file 1: A randomized clinical trial of 4 months Rifampin} vs. 9 months Isoniazid for latent TB infection. - Phase 3 effectiveness.

\section{Abbreviations}

WHO: World health organization; TB: Tuberculosis; LTBI: Latent TB infection; TST: Tuberculin skin test; IGRA: Interferon gamma release assay; QFT-G: QuantiFERON-TB ${ }^{\oplus}$ Gold; NTM: Non-tuberculosis mycobacterium; AFB: Acid-fast bacilli; BCG: Bacillus calmette-guerin; PPD: Purified protein derivative

\section{Competing interests}

All authors certify that there is no conflict of interest with any financial organization regarding the material discussed in the manuscript. The research was funded by Canadian Institutes of Health Research, NCT00170209.

\section{Authors' contributions}

HCK and SJL are guarantors of the manuscript. H.C. Kim: contributed as the corresponding author, reviewing all data, and revising the manuscript. SJL: contributed as the primary author, reviewing all data, and writing the manuscript. YEK: contributed to the preparation of the manuscript, and read and approved the final manuscript. YJC: contributed to the preparation of the manuscript, and read and approved the final manuscript. YYJ: contributed to the preparation of the manuscript, and read and approved the final manuscript. JDL: contributed to the preparation of the manuscript, and read and approved the final manuscript. JRK: contributed to the preparation of the manuscript, and read and approved the final manuscript. YSH: contributed to the preparation of the manuscript, and read and approved the final manuscript. HJK: contributed to the preparation of the manuscript, and read and approved the final manuscript. DM: contributed to the preparation of the manuscript, and read and approved the final manuscript. All authors read and approved the final manuscript.

\section{Acknowledgements}

All authors certify that there is no role of funding body in the collection, analysis, and interpretation of data; in the writing of the manuscript; and in the decision to submit the manuscript for publication.

\section{Author details}

${ }^{1}$ Department of Internal Medicine, College of Medicine, Gyeongsang National University, 90 Chilam-Dong, Jinju, Gyeongnam 660-302, South Korea. ${ }^{2}$ Gyeongsang Institute of Health Sciences, Gyeongsang National University, Jinju, South Korea. ${ }^{3}$ Department of Preventive Medicine, College of Medicine, Gyeongsang National University, Jinju, South Korea. ${ }^{4}$ Korean Institute of Tuberculosis, Seoul, South Korea. ${ }^{5}$ Montreal Chest Institute, McGill University, Montreal, Canada.

Received: 2 July 2013 Accepted: 16 October 2014

Published online: 18 November 2014

\section{References}

1. WHO: Global tuberculosis report. 2013, http://www.who.int/tb/ publications/global_report/en/index.html.

2. The global plan to stop TB, 2006-2015: summary. Wkly Epidemiol Rec 2006, 81(9):86-88.

3. Global tuberculosis control: key findings from the December 2009 WHO report. Wkly Epidemiol Rec 2010, 85(9):69-80.

4. Mazurek GH, Jereb J, Vernon A, LoBue P, Goldberg S, Castro K: Updated guidelines for using interferon gamma release assays to detect mycobacterium tuberculosis infection - United States, 2010. MMWR 2010, 59(RR-5):1-25.

5. Moon H, Lee J: Korean Guidelines for Tuberculosis. Korea: Joint Committee for the Development of Korean Guidelines for Tuberculosis; 2011.

6. Mazurek GH, LoBue PA, Daley CL, Bernardo J, Lardizabal AA, Bishai WR lademarco MF, Rothel JS: Comparison of a whole-blood interferon gamma assay with tuberculin skin testing for detecting latent mycobacterium tuberculosis infection. JAMA 2001, 286(14):1740-1747.
7. Moran-Mendoza O, Marion SA, Elwood K, Patrick D, FitzGerald JM: Risk factors for developing tuberculosis: a 12-year follow-up of contacts of tuberculosis cases. Int J Tuberc Lung Dis 2010, 14(9):1112-1119.

8. El-Sadr WM, Tsiouris SJ: HIV-associated tuberculosis: diagnostic and treatment challenges. Semin Respir Crit Care Med 2008, 29(5):525-531.

9. Brock I, Weldingh K, Lillebaek T, Follmann F, Andersen P: Comparison of tuberculin skin test and new specific blood test in tuberculosis contacts. Am J Respir Crit Care Med 2004, 170(1):65-69.

10. Mori T, Sakatani M, Yamagishi F, Takashima T, Kawabe $Y$, Nagao K, Shigeto $E_{\text {, }}$ Harada N, Mitarai S, Okada M, Suzuki K, Inoue Y, Tsuyuguchi K, Sasaki Y, Mazurek GH, Tsuyuguchi I: Specific detection of tuberculosis infection: an interferon-gamma-based assay using new antigens. Am J Respir Crit Care Med 2004, 170(1):59-64.

11. Horsburgh CR Jr, Rubin EJ: Clinical practice. Latent tuberculosis infection in the United States. N Engl J Med 2011, 364(15):1441-1448.

12. Marks SM, Taylor Z, Qualls NL, Shrestha-Kuwahara RJ, Wilce MA, Nguyen CH: Outcomes of contact investigations of infectious tuberculosis patients. Am J Respir Crit Care Med 2000, 162(6):2033-2038.

13. Morrison J, Pai M, Hopewell PC: Tuberculosis and latent tuberculosis infection in close contacts of people with pulmonary tuberculosis in low-income and middle-income countries: a systematic review and meta-analysis. Lancet Infect Dis 2008, 8(6):359-368.

14. Diel R, Loddenkemper R, Meywald-Walter K, Gottschalk R, Nienhaus A: Comparative performance of tuberculin skin test, QuantiFERON-TB-gold in tube assay, and T-Spot.TB test in contact investigations for tuberculosis. Chest 2009, 135(4):1010-1018.

15. Huang YW, Shen GH, Lee JJ, Yang WT: Latent tuberculosis infection among close contacts of multidrug-resistant tuberculosis patients in central Taiwan. Int J Tuberc Lung Dis 2010, 14(11):1430-1435.

16. Jo KW, Hong Y, Park JS, Bae IG, Eom JS, Lee SR, Cho OH, Choo EJ, Heo JY, Woo JH, Shim TS: Prevalence of latent tuberculosis infection among health care workers in South Korea: a multicenter study. Tuber Respir Dis 2013, 75(1):18-24.

17. Li J, Munsiff SS, Agerton TB: Prevalence of tuberculin skin test positivity in clinical population in New York City. J Immigr Minor Health 2010, 12(6):816-822.

18. Pareek M, Bond M, Shorey J, Seneviratne S, Guy M, White P, Lalvani A, Kon OM: Community-based evaluation of immigrant tuberculosis screening using interferon gamma release assays and tuberculin skin testing: observational study and economic analysis. Thorax 2013, 68(3):230-239.

19. Shanaube K, Hargreaves J, Fielding K, Schaap A, Lawrence KA, Hensen B, Sismanidis C, Menezes A, Beyers N, Ayles H, Godfrey-Faussett P: Risk factors associated with positive QuantiFERON-TB gold in-tube and tuberculin skin tests results in Zambia and South Africa. PLoS One 2011, 6(4):e18206

20. Mack U, Migliori GB, Sester M, Rieder HL, Ehlers S, Goletti D, Bossink A, Magdorf K, Holscher C, Kampmann B, Arend SM, Detjen A, Bothamley G, Zellweger JP, Milburn H, Diel R, Ravn P, Cobelens F, Cardona PJ, Kan B, Solovic I, Duarte R, Cirillo DM, Lange C: LTBI: latent tuberculosis infection or lasting immune responses to $\mathrm{M}$. tuberculosis? A TBNET consensus statement. Eur Respir J 2009, 33(5):956-973.

21. Kim HJ, Yoon HI, Park KU, Lee CT, Lee JH: The impact of previous tuberculosis history on T-SPOT.TB(R) interferon-gamma release assay results. Int J Tuberc Lung Dis 2011, 15(4):510-516.

22. Lalvani A, Pathan AA, McShane $H$, Wilkinson RJ, Latif M, Conlon CP, Pasvol $G$, Hill AV: Rapid detection of mycobacterium tuberculosis infection by enumeration of antigen-specific T cells. Am J Respir Crit Care Med 2001, 163(4):824-828.

23. Carrara S, Vincenti D, Petrosillo N, Amicosante M, Girardi E, Goletti D: Use of a T cell-based assay for monitoring efficacy of antituberculosis therapy. Clin Infect Dis 2004, 38(5):754-756.

24. Hu Y, Zhao Q, Graviss EA, Jiang W, Yuan Z, Xu B: Use of the T-SPOT.TB assay to screen latent tuberculosis infection among the TB contacts in Shanghai, China. J Infect 2012, 65(1):39-48.

25. Lienhardt C, Fielding K, Sillah J, Tunkara A, Donkor S, Manneh K, Warndorff D. McAdam KP, Bennett S: Risk factors for tuberculosis infection in sub-Saharan Africa: a contact study in the Gambia. Am J Respir Crit Care Med 2003, 168(4):448-455. 
26. Ozturk N, Surucuoglu S, Ozkutuk N, Gazi H, Akcali S, Koroglu G, Cicek C: Comparison of interferon-gamma whole blood assay with tuberculin skin test for the diagnosis of tuberculosis infection in tuberculosis contacts. Mikrobiyol Bul 2007, 41(2):193-202.

27. van Zyl-Smit RN, Pai M, Peprah K, Meldau R, Kieck J, Juritz J, Badri M, Zumla A, Sechi LA, Bateman ED, Dheda K: Within-subject variability and boosting of T-cell interferon-gamma responses after tuberculin skin testing. Am J Respir Crit Care Med 2009, 180(1):49-58.

doi:10.1186/s12879-014-0566-4

Cite this article as: Lee et al:: Risk factors for latent tuberculosis

infection in close contacts of active tuberculosis patients in South

Korea: a prospective cohort study. BMC Infectious Diseases 2014 14:566.

\section{Submit your next manuscript to BioMed Central and take full advantage of:}

- Convenient online submission

- Thorough peer review

- No space constraints or color figure charges

- Immediate publication on acceptance

- Inclusion in PubMed, CAS, Scopus and Google Scholar

- Research which is freely available for redistribution 